\title{
REDESCRIPTION ET ANALYSE CRITIQUE DE QUELQUES ESPECES D'OXYURES \\ DE LA TORTUE GRECQUE (TESTUDO GRAECA L.) DIVERSITE DES STRUCTURES CEPHALIQUES (II)
}

\author{
Par Annie-J. PETTER
}

Nous avons, dans une récente publication, donné la description, accompagnée d'une étude détaillée des structures céphaliques, de sept espèces d'Oxyures de la Tortue grecque. Nous décrivons ici quatre autres espèces que l'on rencontre habituellement dans le cæcum des Tortues terrestres.

Les spécimens étudiés ont été recueillis dans les cæcums de Tortues grecques (Testudo graeca L.), ramassées dans les régions de Chénoua et d'Aumale (Algérie), ainsi que chez deux Tortues grecques de provenance inconnue.

\section{I. - Tachygonetria longicollis (Schneider) (fig. 1).}

Synon. : Oxyuris longicollis Schneider 1866; Paracis longicollis Railliet et Henry 1916; Tachygonetria setosa Seurat 1918; Tachygonetria massinissae Seurat 1918 ; Tachygonetria pusilla Seurat 1918; Tachygonetria longicollis $q$ sensu Thapar 1925, nec ơ sensu Thapar 1925 ; Tachygonetria pusilla ô sensu Thapar 1925, nec $q$ sensu Thapar 1925 ; Tachygonetria testudinis Forstner 1960.

\section{Description}

Espèce à œsophage long, à extrémité apicale plate et non conique, ce qui la différencie à première vue de Tachygonetria conica.

Bouche triangulaire, munie de trois lèvres. Deux amphides latérales, et quatre petites papilles submédianes, en position très périphérique en vue apicale. Cycle interne de six papilles, deux par lèvre. La cuticule de l'extrémité apicale est épaissie suivant des lignes formant des dessins sinueux, avec quatre anses principales correspondant aux quatre axes submédians.

Esophage très long et mince, avec corpus et isthme terminé par un bulbe valvulé. L'extrémité antérieure de l'œsophage est munie de trois grosses dents dressées portées par de courts pédicules. Chez la femelle, la partie antérieure de l'intestin est dilatée en forme de massue. 


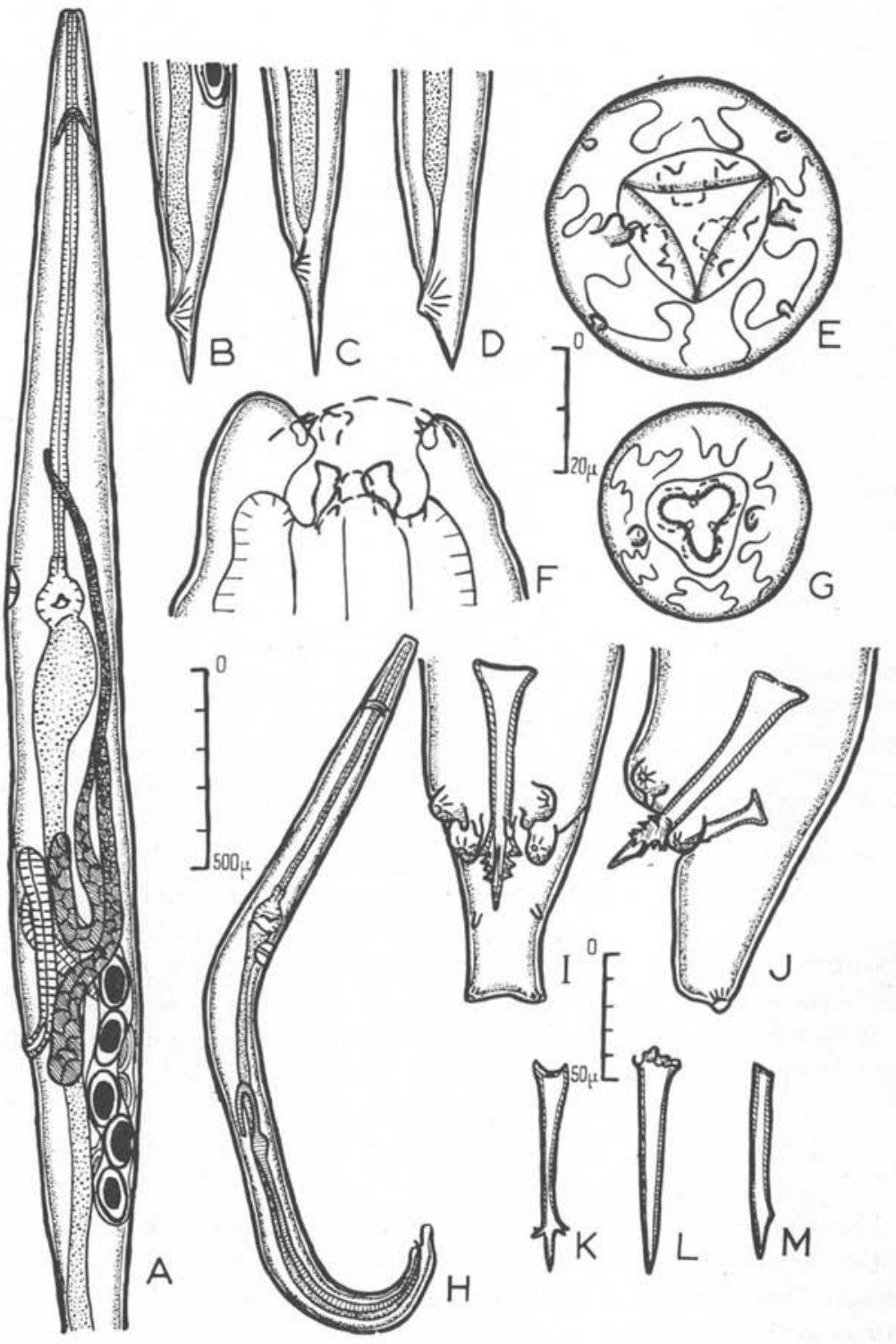

Fig. 1. - Tachygonetria longicollis. A) Femelle, portion antérieure du corps, vue latérale. - B) Femelle, extrémité postérieure, vue latérale. - C) et D) Femelles, extrémités postérieures de deux autres spécimens, vues latérales. E) Femelle, vue apicale. - F) Femelle, extrémité antérieure, vue latérale (tes traits en pointillé sont les traits de surface). - G) Mâle, vue apicale. H) Mâle, vue latérale. - I) Mâle, extrémité postérieure, vue ventrale. J) Mâle, extrémité postérieure, vue latérale. - K) Spicule d'un autre spécimen, vue ventrale. - L) et M) Spicule d'un autre spécimen, vue ventrale (L) et latérale (M). (Fig. A, B, C, D, H : échelle 0-500 u ; fig. E, F, G : échelle 0-20 u ; fig. I, J, K, L, M : échelle 0-50 «). 
Femelle : Les femelles mesurent de $3 \mathrm{~mm}$. à $4,5 \mathrm{~mm}$. environ. Nous donnons les principales mensurations d'une femelle de $3,68 \mathrm{~mm}$. : largeur maximum : $300 \mu$; anneau nerveux, pore excréteur et vulve respectivement à $250 \mu$, $1,42 \mathrm{~mm}$. et $2,56 \mathrm{~mm}$. de l'extrémité antérieure ; longueur de l'œsophage : $1,5 \mathrm{~mm}$. ; longueur de la queue : $160 \mu$; œufs de $130 \mu$ sur $60 \mu$.

La longueur de la queue varie légèrement d'un spécimen à l'autre ; chez certains grands spécimens de plus de $4 \mathrm{~mm}$., elle est très courte $(150 \mu)$ et de forme conique, à pointe pas du tout effilée ; chez d'autres spécimens, elle peut atteindre $260 \mu$.

L'appareil génital présente la structure habituelle du genre Tachygonetria.

Mâle : Les mâles mesurent de 2 à $2,8 \mathrm{~mm}$. environ. Nous donnons les principales mensurations d'un spécimen de 2,2 mm. : largeur maximum : $120 \mu$; anneau nerveux et pore excréteur respectivement à $180 \mu$ et $860 \mu$ de l'extrémité antérieure ; longueur de l'œsophage : $800 \mu$; longueur de la queue : $60 \mu$; longueur du spicule : $100 \mu$.

Queue dorsale de $60 \mu$, tronquée à l'extrémité, et portant une paire de papilles latérales aux angles de l'extrémité tronquée. Phasmides à $30 \mu$ de l'extrémité. Trois paires de papilles au niveau du cloaque; la paire pré-cloacale est arrondie et munie d'un petit lobe postérieur. La lèvre inférieure du cloaque se prolonge en un mamelon tronconique impair, dans lequel s'engage l'extrémité d'un gubernaculum en forme de $\mathrm{V}$ très ouvert.

Spicule long de $80 \mu$ à $100 \mu$, souvent exserti en partie. L'extrémité du spicule est généralement barbelée, le développement dès barbillons étant variable d'un individu à l'autre : chez certains, ils semblent manquer complètement, l'extrémité du spicule étant simplement taillée en biseau ; chez d'autres, il existe une paire de barbillons qui donnent à l'extrémité l'aspect de la fleur de lis ; chez certains, enfin, il existe deux ailes attachées à peu de distance de l'extrémité, et dont le bord libre est en dent dé scie.

\section{Discussion}

L'espèce peut être identifiée à Tachygonetria longicollis (Schneider).

Les dimensions indiquées par Schneider concordent avec celles de notre espèce ; Drasche donne des longueurs plus faibles, mais l'aspect de la vue apicale et de la queue du mâle sont identiques aux nôtres.

Dans son tableau des espèces africaines de Tachygonetria, Seurat distingue quatre espèces possédant une tête hémisphérique avec trois lèvres semi-circulaires peu élevées: $T$. setosa, $T$. massinissae, $T$. pusilla et $T$. longicollis.

Tachygonetria setosa ne diffère de Tachygonetria longicollis que par sa pilosité plus abondante, et les deux espèces ont été mises en synonymie par Thapar. 
Seurat différencie les trois autres espèces par la longueur de la queue chez la femelle et la forme du spicule chez le mâle (présence ou absence d'une ou plusieurs paires de barbillons). Nous avons constaté que ces deux caractères étaient variables dans les limites indiquées par Seurat, pour des spécimens à part cela identiques, et présentant en particulier des vues apicales semblables ; les quatre espèces de Seurat nous semblent donc devoir être mises en synonymie.

Thapar décrit deux espèces différentes sous les noms de Tachygonetria pusilla et Tachygonetria longicollis; comme nous l'avons vu dans une précédente publication (Petter, 1961), sa figure représentant la queue du mâle de Tachygonetria longicollis correspond plutôt à la queue du mâle de Tachygonetria conica; d'autre part, il décrit pour Tachygonetria pusilla une femelle à queue très longue : $560 \mu$, ce qui ne correspond pas à l'espèce de Seurat, mais plutôt à la femelle de Tachygonetria macrolaimus; enfin, sa description du mâle de Tachygonetria pusilla correspond à celle du mâle de Tachygonetria longicollis au sens de Seurat; il semble donc que son espèce $T$. pusilla ne peut être retenue.

Dans un récent travail, Forstner (1960) crée une nouvelle espèce, Tachygonetria testudinis, très proche de Tachygonetria longicollis, mais en différant par une taille plus grande, un œsophage plus court, et une queue et un spicule plus courts chez le mâle. Cependant, les photos qui accompagnent le texte montrent qu'il a travaillé sur du matériel fixé, et ayant dans certains cas subi une contraction qui entraîne un raccourcissement du corps et de l'appendice caudal, alors que l'œsophage plus rigide conserve sa longueur. Les figures qu'il donne des mâles des deux espèces ne montrent aucune différence nette dans la forme du spicule; il nous semble donc que Tachygonetria testudinis doit également être mis en synonymie avec Tachygonetria longicollis.

\section{II. - Tachygonetria robusta (Drasche) (fig. 2).}

Synon: Oxyuris robusta Drasche 1883; Oxyuris draschei Stossich i898; Mehdiella microstoma Seurat 1918 pro parte; Tachygonetria microstoma Thapar 1925 pro parte.

\section{Description}

Espèce à corps robuste, à tête large et à queue courte.

Femelle : Femelles de grande taille (7 à 7,6 mm.), à tête très grosse $(150 \mu$ de diamètre).

Ouverture buccale triangulaire, munie de trois lèvres transparentes, mais dont le plan de symétrie ne coïncide pas avec le plan de symétrie du corps; il existe une grande lèvre latéro-ventrale gauche rectangulaire et deux lèvrès plus petites et à bords arrondis, en position respectivement latéro-dorsale et 


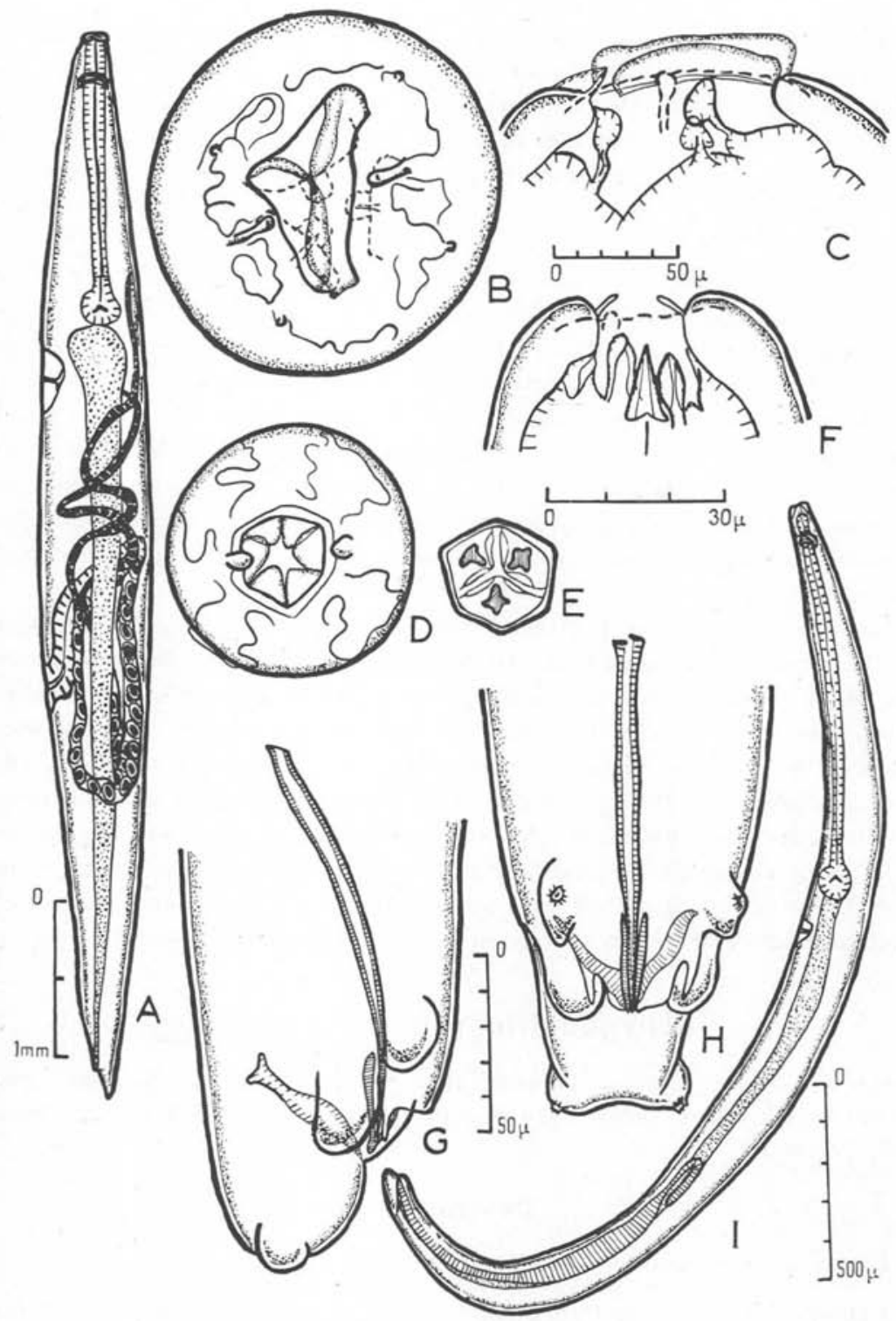

Fig. 2. - Tachygonetria robusta. A) Femelle, vue latérale. - B) Femelle, vue apicale. - C) Femelle, extrémité antérieure, vue latérale (les traits en pointillé sont les traits de surface). - D) Mâle, vue apicale. - E) Mâle, section transversale de la cavité buccale. - F) Mâle, extrémité antérieure, vue latérale (les traits en pointillé sont les traits de surface). - G) Mâle, extrémité postérieure, vue latérale. - H) Mâle, extrémité postérieure, vue ventrale. I) Mâle, vue latérale. 
latéro-ventrale droites. Deux amphides latérales et quatre petites papilles submédianes. La cuticule de l'extrémité apicale est épaissie suivant des lignes sinueuses qui encadrent les amphides et les papilles submédianes.

Esophage court relativement à la grande taille du corps, avec corpus qui semble divisé en deux parties par une ligne de discontinuité, et isthme terminé par un bulbe valvulé. L'extrémité antérieure de l'œsophage est munie de trois grosses dents dressées à l'extrémité de minces pédicules.

Nous donnons les mensurations d'un spécimen de $7 \mathrm{~mm}$.: largeur maximum : $700 \mu$; anneau nerveux, pore excréteur et vulve respectivement à $260 \mu$, $2,3 \mathrm{~mm}$. et $4,25 \mathrm{~mm}$. de l'extrémité antérieure; longueur de l'œsophage: $1,9 \mathrm{~mm}$. ; longueur de la queue : $200 \mu$; dimensions des œufs : $180 \mu$ sur $90 \mu$. La dimension des œufs est variable avec les spécimens : chez un spécimen de $7,6 \mathrm{~mm}$., ils mesurent seulement $110 \mu$ sur $70 \mu$.

Ovéjecteur du type habituel, utérus parallèles dirigés vers l'extrémité antérieure, ovaires remontant le long de l'intestin en décrivant plusieurs boucles.

MIâle: Les mâles mesurent environ $3 \mathrm{~mm}$.

L'ouverture buccale est hexagonale et munie de six lèvres transparentes coniques. Deux amphides latérales et quatre petites papilles submédianes périphériques. La cuticule de l'extrémité apicale est épaissie suivant des lignes formant quatre anses submédianes à contours sinueux.

Esophage long et mince, avec corpus et isthme terminé par un bulbe valvulé. L'extrémité antérieure de l'œsophage est munie de trois dents dressées de structure complexe : avec un axe chitinoïde et des bords membraneux.

Nous donnons les mensurations d'un spécimen de $2,88 \mathrm{~mm}$. : largeur maximum : $170 \mu$; anneau nerveux et pore excréteur respectivement à $100 \mu$ et 1,1 mm. de l'extrémité antérieure ; longueur de l'œsophage : $1 \mathrm{~mm}$. ; longueur de la queue : $30 \mu$; longueur du spicule : $110 \mu$.

Queue dorsale très courte et globuleuse, tronquée à l'extrémité et portant une paire de papilles latérales aux angles de l'extrémité tronquée. Trois paires de papilles au niveau du cloaque. Gubernaculum en forme de V très ouvert. Spicule aciculaire, étroit et allongé. Son extrémité est entourée par un étui chitinoïde à paroi dorsale bien développée et haute de $28 \mu$, tandis que sa paroi ventrale est beaucoup plus courte et mince.

\section{Discussion}

Nous avons rapporté les mâles aux femelles dans cette espèce en tenant compte de l'aspect massif du corps et de la queue très courte dans les deux sexes, mais nous voyons que l'aspect de leur extrémité antérieure est complètement différent : alors que le mâle présente une ouverture buccale hexagonale, type considéré comme primitif, la femelle a au contraire une structure buccale très évoluée ; il s'est produit, comme chez Mehdiella microstoma, une torsion 
de l'extrémité antérieure, mais moins poussée que chez cette espèce : la lèvre dorsale a changé d'orientation, tendant à occuper une position latéro-dorsale droite, et la lèvre ventro-latérale gauche s'est allongée, mais est encore en position latéro-ventrale : nous avons donc une structure intermédiaire entre la structure typique à symétrie triradiée et la structure à deux lèvres latérales de Mehdiella microstoma (voir fig. 3).

L'espèce peut être identifiée à Tachygonetria robusta (Drasche). Drasche décrit uniquement les femelles, que Seurat et Thapar identifient aux femelles de Mehdiella microstoma (Drasche).

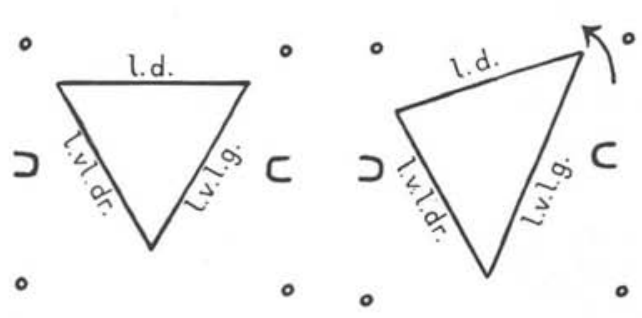

A

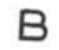

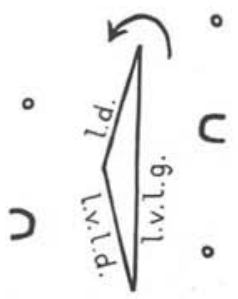

o

Fıg. 3. - Schéma montrant l'évolution des structures céphaliques chez Tachygonetria robusta et Mehdiella microstoma. A) Structure à symétric triradiée. - B) Structure de Tachygonetria robusta. - C) Structure de Mehdiella microstoma (l.d. : lèvre dorsale ; 1. v.l. dr. : lèvre ventro-latérale droite; 1 . v.l. g. : lèvre ventro-latérale gauche).

Nous pensons avec Dubinina que les caractères indiqués par Drasche ne correspondent pas parfaitement à ceux de la femelle de Mehdiella microstoma (en particulier par la position du pore excréteur, qui est nettement en arrière du bulbe, alors que, chez Mehdiella microstoma, il est en avant du bulbe). Dubinina donne une description de la femelle et du mâle de Tachygonetria robusta, accompagnée de figures qui correspondent parfaitement aux nôtres, sa vue apicale de la femelle étant sans doute en réalité une vue profonde correspondant à une section œsophagienne.

\section{III. - Tachygonetria microlaimus (Linstow) (fig. 4).}

Synon. : Oxyuris microlaimus of sensu Linstow 1899, nec + sensu Linstow 1899 ; Tachygonetria microlaimus ô sensu Thapar 1925, nec + sensu Thapar 1925.

\section{Description}

Espèce de grande taille, à corps robuste. 

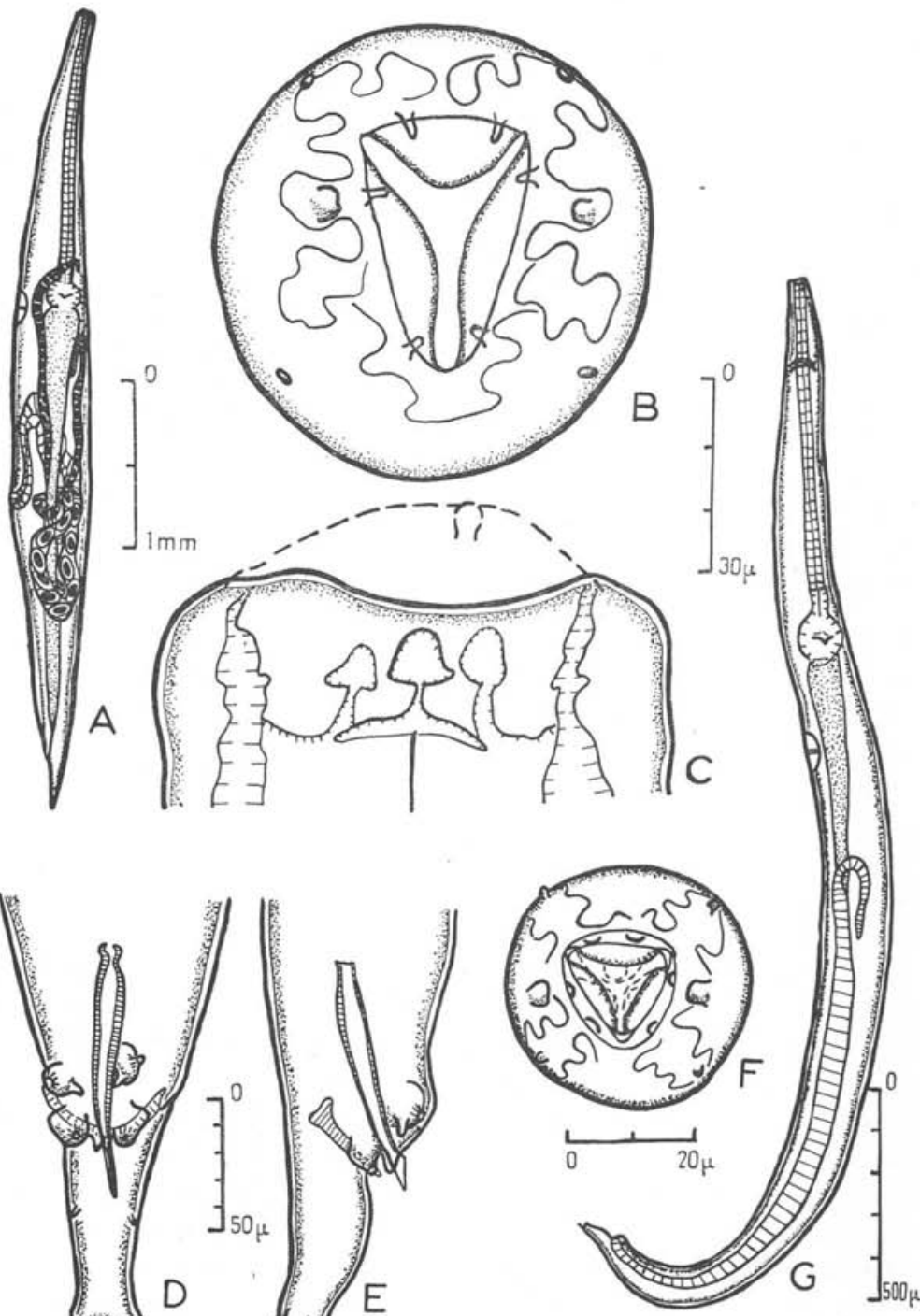

Fıg. 4. - Tachygonetriu microlaimus. A) Femelle, vue latérale. - B) Femelle, vue apicale. - C) Femelle, extrémité antérieure, vue latérale (les traits en pointillé sont les traits de surface). - D) Mâle, extrémité postérieure, vue ventrale. - E) Mâle, extrémité postérieure, vue latérale. - F) Mâle, vue apicale. - G) Mâle, vue latérale. 
Femelle : Les femelles mesurent de 3,5 à $5 \mathrm{~mm}$. environ. La tête est large (70 à $80 \mu$ de diamètre). L'ouverture buccale est triangulaire, avec un petit côté dorsal et deux grands côtés latéro-ventraux. Elle est munie de trois lèvres transparentes. Deux amphides légèrement déplacées vers la face ventrale et quatre petites papilles submédianes périphériques. Cycle interne de six papilles, deux par lèvre. La cuticule de l'extrémité apicale est épaissie suivant des lignes qui forment des dessins sinueux.

Esophage long et mince, avec corpus et isthme terminé par un bulbe valvulé. L'extrémité antérieure de l'œsophage est munie de trois grosses dents dressées portées à l'extrémité de minces pédicules.

Nous donnons les principales mensurations d'un spécimen de 5,15 mm.: largeur maximum : $450 \mu$; anneau nerveux, pore excréteur et vulve respectivement à $250 \mu, 2 \mathrm{~mm}$. et $3,2 \mathrm{~mm}$. de l'extrémité antérieure ; longueur de l'œsophage : $2 \mathrm{~mm}$. ; longueur de la queue : $350 \mu$; œufs de $180 \mu$ sur $80 \mu$.

Ovéjecteur du type habituel, avec sphincter dilaté en ampoule à son extrémité. Utérus parallèles dirigés vers l'avant; ovaires remontant vers l'avant jusqu'au niveau du bulbe.

Mâle: Les mâles mesurent de 2,4 à $3 \mathrm{~mm}$.

Ouverture buccale triangulaire munie de trois lèvres transparentes rectangulaires. Deux amphides latérales légèrement déplacées vers la face ventrale et quatre petites papilles submédianes en position périphérique. Cycle interne de six papilles, deux par lèvre. La cuticule de l'extrémité apicale est épaissie suivant des lignes qui dessinent quatre anses submédianes à contours sinueux.

Esophage long et mince, avec corpus et isthme terminé par un bulbe valvulé. L'extrémité antérieure de l'œsophage est munie de trois grosses dents dressées sur de très courts pédicules.

Nous donnons les principales mensurations d'un mâle de $2,82 \mathrm{~mm}$. : largeur maximum : $160 \mu$; anneau nerveux et pore excréteur respectivement à $200 \mu$ et $1,13 \mathrm{~mm}$. de l'extrémité antérieure ; longueur de l'œsophage : $900 \mu$; longueur de la queue : $70 \mu$; longueur du spicule : $90 \mu$.

Queue dorsale longue et légèrement arquée à concavité dorsale ; son extrémité est tronquée et munie d'une fine pointe hyaline de $10 \mu$. Une paire de grosses papilles latérales aux angles de l'extrémité tronquée ; trois paires de papilles au niveau du cloaque ; la paire précloacale est arrondie et munie d'un petit lobe postérieur. La lèvre inférieure du cloaque se prolonge en un mamelon tronconique où s'engage la pointe d'un gubernaculum en $\mathrm{V}$ très ouvert. Spicule aciculaire, long d'environ $90 \mathrm{p}$, à pointe taillée en biseau.

\section{Discussion}

Linstow (1899) crée une espèce nouvelle : Oxyuris microlaimus, dont le mâle correspond au nôtre par les dimensions et les caractères morphologiques, en particulier la fine pointe hyaline de l'extrémité caudale. Par contre, la femelle 
ne peut correspondre à la nôtre à cause de son œsophage court $(1 / 8$ de la longueur du corps, alors que, chez notre espèce, il mesure $1 / 2,5$ de cette longueur).

Thapar donne une description de Tachygonetria microlaimus, dont le mâle peut également correspondre au nôtre, tandis que la femelle, par sa taille $(3 \mathrm{~mm}$.) et la queue très courte $(170 \mu)$, correspondrait plutôt à la femelle de Tachygonetria longicollis (elle ne peut correspondre à la femelle de l'Oxyuris microlaimus de Linstow à cause de la longueur de son œsophage).

Les femelles et les mâles décrits par nous se sont rencontrés seulement chez certaines des tortues autopsiées, où ils sont toujours associés; nous les avons appariés en tenant compte de ce fait, mais, l'extrémité apicale ayant un aspect différent dans les deux sexes, nous ne pouvons affirmer qu'il s'agit de la même espèce. Nous pensons, cependant, que cela est probable et, en raison de la similitude du mâle avec celui de Linstow, nous appelons l'espèce Tachygonetria microlaimus.

\section{IV. - Mehdiella stylosa (Thapar) (fig. 5).}

Synon. : Tachygonetria stylosa $q$ sensu Thapar 1925, nec \& sensu Thapar 1925 ; Tachygonetria stylosa Dubinina 1949.

\section{Description}

Espèce à œsophage long, caractérisée chez la femelle par une tête munie de 12 petits stylets, chez le mâle par une queue retroussée dorsalement.

F'emelle : Les femelles mesurent de 5 à $6 \mathrm{~mm}$. La paroi du corps est légèrement comprimée et détachée de la cuticule en arrière de la tête, formant une très légère vésicule céphalique.

Ouverture buccale dessinant un triangle à angles arrondis, dont chaque côté présente deux ondulations. Elle est munie de trois lèvres transparentes. Deux amphides latérales et quatre petites papilles submédianes en position périphérique; de plus, il existe 12 petites languettes dressées faisant saillie au-dessus de l'ouverture buccale et disposées en cercle. Le parenchyme du corps se prolonge dans ces languettes qui sont groupées deux par deux sur une base parenchymateuse commune. Sans doute s'agit-il des six papilles du cycle interne modifiées et dédoublées. La cuticule de l'extrémité apicale est épaissie suivant des lignes qui dessinent quatre anses submédianes trilobées et deux anses médianes simples.

Esophage long et mince, avec corpus et isthme terminé par un bulbe valvulé. L'extrémité antérieure de l'œsophage est munie de trois grosses dents dressées et portées par de courts pédicules.

Nous donnons les mensurations d'un spécimen de 4,96 mm. : largeur maximum : $300 \mu$; anneau nerveux, pore excréteur et vulve respectivement à $200 \mu$, 


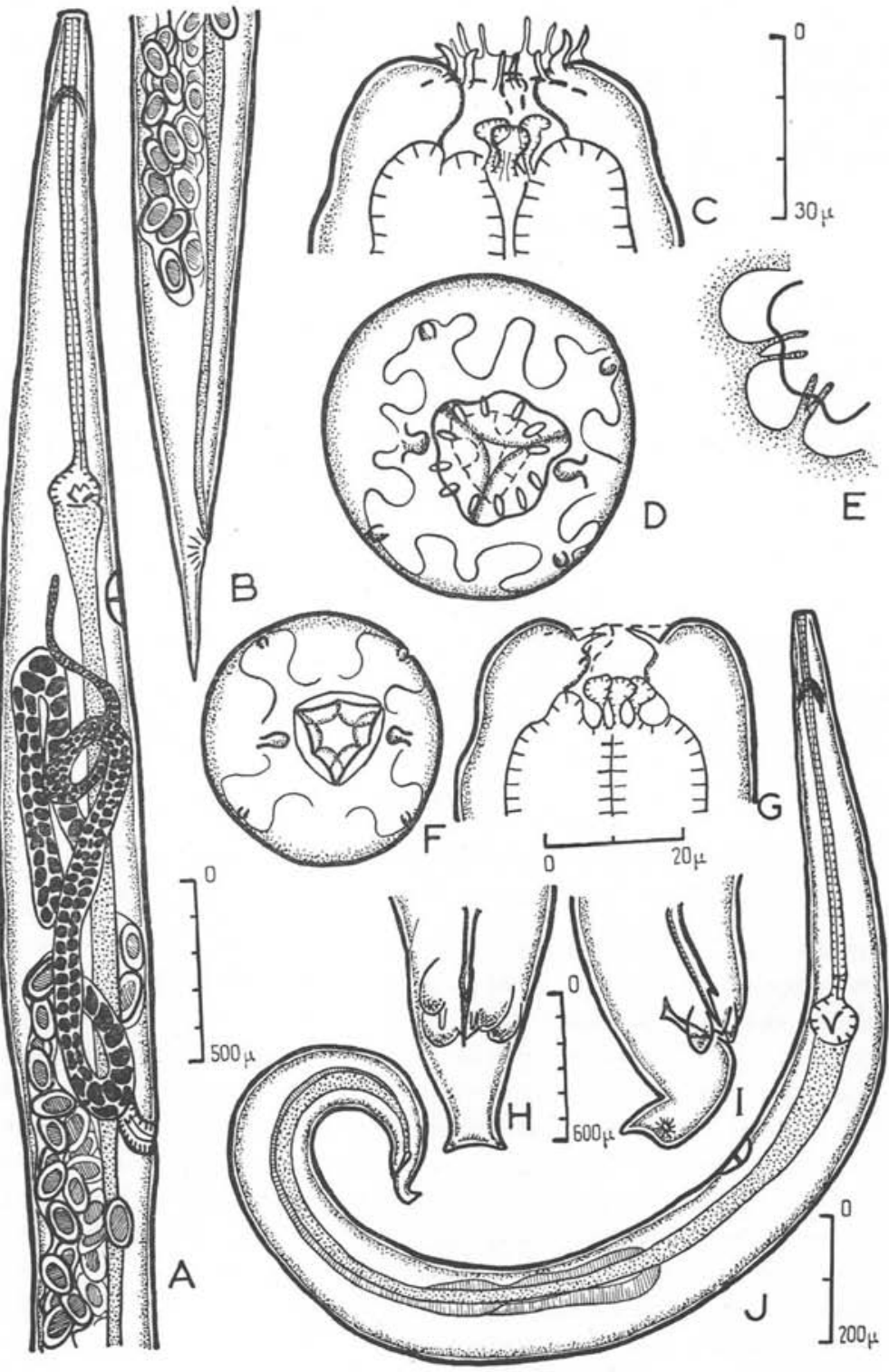

Fig. 5. - Mehdiella stylosa. A) Femelle, portion antérieure, vue latérale. B) Femelle, portion postérieure, vue latérale. - C) Femelle, extrémité antérieure, vue latérale (les traits en pointillé sont les traits de surface). D) Femelle, vue apicale. - E) Femelle, extrémité antérieure, vue apicale légèrement oblique. - F) Mâle, vue apicale. - G) Mâle, extrémité antérieure, vue latérale (les traits en pointillé sont les traits de surface). - H) Mâle, extrémité postérieure, vue ventrale. - I) Mâle, extrémité postérieure, vue latérale. - J) Mâle, vue latérale. (Fig. C, D, E, F : échelle 0-30 $\mu$; fig. G : échelle 0-20 $u$ ). 
1,55 mm. et 2,97 mm. de l'extrémité antérieure ; longueur de l'œsophage : $1,3 \mathrm{~mm}$.; longueur de la queue : $350 \mu$; œufs de $110 \mu$ sur $70 \mu$.

Ovéjecteur du type habituel, utérus parallèles et dirigés vers l'extrémité antérieure, ovaires remontant le long de l'intestin en décrivant des boucles.

Mâle : L'extrémité apicale du mâle est complètement différente de celle de la femelle, et nous n'avons pas observé les languettes caractéristiques de celle-ci. L'ouverture buccale est hexagonale, avec tendance à la forme triangulaire ; elle est munie de six petites lèvres arrondies. Deux amphides latérales et quatre petites papilles submédianes périphériques. La cuticule de l'extrémité apicale est épaissie suivant des lignes qui dessinent quatre anses submédianes.

Esophage long et mince, avec corpus et isthme terminé par un bulbe valvulé. L'extrémité antérieure de l'œsophage est munie de trois dents dressées sur de courts pédicules.

Nous donnons les mensurations d'un spécimen de 2,37 mm. : largeur maximum : $150 \mu$; anneau nerveux et pore excréteur respectivement à $100 \mu$ et $950 \mu$ de l'extrémité antérieure ; longueur de l'œsophage : $700 \mu$; longueur de la queue : $40 \mu$; longueur du spicule : $55 \mu$.

La queue, courte, est retroussée dorsalement et munie d'une paire de papilles latérales à l'extrémité. Trois paires de papilles au niveau du cloaque. Gubernaculum en $\mathrm{V}$ très ouvert. Spicule court et muni d'une épine ventrale à peu de distance de son extrémité.

\section{Discussion}

L'espèce peut être identifiée à Mehdiella stylosa (Thapar) par l'aspect caractéristique des petites languettes dressées de son extrémité apicale. Les femelles décrites par Thapar correspondent parfaitement aux nôtres, mais les mâles sont complètement différents.

Dubinina décrit des mâles dont les caractères sont identiques aux nôtres, bien que les mesures de la queue et du spicule soient légèrement supérieures. Cependant, elle indique que les lèvres du mâle possèdent les mêmes stylets que celles de la femelle, alors que nous ne les avons jamais observés sur nos mâles, qui possèdent une structure apicale à six lèvres complètement différente de celle des femelles; nous avons fait l'attribution surtout par élimination, mais peut-être existe-t-il d'autres mâles rares que nous n'avons pas rencontrés et qui présentent le même aspect apical que les femelles.

Forstner crée deux espèces nouvelles, Mehdiella hamosa et Mehdiella cordata, dont il n'a pas rencontré les femelles. Il différencie Mehdiella cordata de la précédente par l'aspect de la queue qui est divisée en deux parties par un étranglement et se termine en une pointe coniqué. Il nous semble que cet étranglement est un artefact dû à la fixation et que les deux espèces n'en font en réalité qu'une seule, dont la description correspond parfaitement au mâle que nous avons attribué à Mehdiella stylosa. 


\section{RÉSUMÉ}

Nous donnons la description, accompagnée d'une étude détaillée des structures céphaliques, de quatre espèces d'Oxyures parasites du cæcum de la Tortue grecque (Testudo graeca L.) :

$1^{\circ}$ Tachygonetria longicollis (Schneider): Tachygonetria pusilla Seurat et Tachygonetria testudinis Forstner sont mis en synonymie avec cette espèce.

$2^{\circ}$ Tachygonetria robusta (Drasche) : L'espèce présente un cas de dimorphisme sexuel de la région antérieure, le mâle ayant une bouche primitive à six lèvres, alors que la femelle a une structure buccale très évoluée.

$3^{\circ}$ Tachygonetria microlaimus (Linstow) : Nous attribuons aux mâles de l'espèce décrite par Linstow des femelles qui ne correspondent pas aux siennes.

$4^{\circ}$ Mehdiella stylosa (Thapar): En accord avec Dubinina, nous attribuons aux femelles de l'espèce créée par Thapar des mâles qui ne correspondent pas à ceux de sa description. Mehdiella hamosa Forstner et Mehdiella cordata Forstner sont mis en synonymie avec le mâle de Mehdiella stylosa.

Les deux espèces précédentes présentent également des cas de dimorphisme sexuel de la région antérieure.

\section{BiBLIOGRAPHIE}

Drasche (R.), 1884. - Nematoden aus Testudo graeca. Verhandl. d.k.k. Zool. Bot. Gesellsch. Wicn, Bd. 33, SS. 325-330.

Dubinina (M. H.), 1949. - Ekologitcheskoïe issledovanie parasitofauni stepnoi tcherepachi (Testudo horsfieldi Gray) Tadjikistana. Parasit. Sb. Zool. Inst. A.N. S.S.S.R., XI, 61-97.

Forster (M. J.), 1960. - Ein Beitrag zur Kenntnis parasitischer Nematoden aus griechischen Landschildkröten. Z. f. Parasitenkunde, 20, 1-22.

Lisstow (O.), 1899. - Nematoden aus der Berliner Zoologischen Sammlung. Mitt. aus d. Zool. Samml. d. Mus. Natur., Berlin, Bd. 1, H. 2, SS. 3-28.

Petter (A.-J.), 1961. - Redescription et analyse critique de quelques espèces d'Oxyures parasites du c. cum de la Tortue grecque (Testudo graeca L.). Diversité des structures céphaliques. Ann Parasit., XXXVI, n ${ }^{\circ} 4$, pp. 648-671.

Schneider (A.), 1856. - Monographie der Nematoden, Berlin, xin + 357 SS.

Seurat (L.-G.), 1918. - Contribution à l'étude de la faune parasitaire de la Tunisie. Arch. de l'Inst. Pasteur de Tunis, t. 10, n 4, pp. 243-275.

Thapar (G. S.), 1925. - Studies on the Oxyurid Parasites of Reptiles. Journ. Helm., v. $3, \mathrm{n}^{\circ} 3-4$, pp. 83-150.

\section{Muséum National d'Histoire Naturelle}

Laboratoire de Zoologie (Vers) (Directeur: A.-G. Chaвaud) et Laboratoire d'Ethologie des Animaux Sauvages (Directeur: J. NouvEL) 